\title{
Study DNA Damage after Photodynamic Therapy using Silver Nanoparticles with A549 cell line
}

\section{El-Hussein A*}

The National Institute of Laser Enhanced Science, Cairo University, Egypt

\begin{abstract}
Lung Cancer is considered one of the major health problems worldwide. Nanomedicine is considered one of the promising research applications nowadays. This is due to the unique physical and chemical properties of the nanoscale elements. Silver nanoparticles (Ag NPs) in specific has been extensively studied recently in many biomedical applications especially in cancers, since they possess multifunctional effects that make these nanostructures ideal candidates for biomedical applications. Ag NPs were proved to have anti- tumor activity with apoptotic cell death pathway. The goal of the current work was to investigate the degree of DNA damage that results from the usage of Ag NPs as a photosensitizer (PS) in photodynamic therapy (PDT), besides to evaluate the reactive oxygen species (ROS) yield that was associated. The results showed the occurrence of DNA damage in lung cancer cells (A549) through the generation of ROS via the detection of the mitochondrial membrane potential changes.
\end{abstract}

Keywords: Silver nanoparticles; Reactive oxygen species; DNA damage; Mitochondria membrane potential

\section{Introduction}

Lung cancer is considered one of the main death cause in all over the world now, not only in the western portion of the world. It is believed to be the major account for about 1.6 million deaths, $20 \%$ of the total cancer deaths [1]. The effect of this disease has increased lately in Africa due to the tobacco epidemic that resulted in its vast dispersion of this disease [2]. According to the international agency for research on cancer (IARC), smoking alone is attributing for about $65 \%$ of lung cancer. Their estimations extend to the appearance of 1.8 million new cases in 2012 where about $60 \%$ of this number is only in the less developing countries [3]. $85 \%$ of lung cancer patients are diagnosed with either metastasis or advanced local tumors or both. Surgery could be one of the first approaches, but unfortunately it is only valid for $15 \%$ of the patients. Bronchoscopic therapy could be the quickest choice and even the only possible choice in treating superficial tumors that are found intraluminally and for the treatment of tumors in the main airways as it can cause a rapid symptomatic relief for those patients and hence their overall conditions. This can give the chance for the chemo and/or radio therapeutic treatments to maintain or improve local patients' conditions [4]. The blockage of the main airways by tumors are the main cause of deaths due to dyspnoea and obstructive pneumonia. However, this approach as well fails with deep tumors and those who are extension distal to the segmental bronchi. The term tumor resistance is very familiar since high proportion of lung cancers were found to be resistant to conventional combined therapies that eventually can't compete with will tumor recurrence or progression [5]. Thus there was an urgent need to introduce a new approach like photodynamic therapy (PDT) for the treatment of lung cancer disease. More than quarter of a century for the active application of PDT in the oncology field to gain somehow a significant clinical applications for either cancer treatment or skin related diseases. It was believed for this kind of new treatment at that time to cause an outbreak in the treatment protocols due to its selectivity and thanks to the high photosensitizer (PS) affinity to tumor tissue, and the local illumination of the tumor area and finally to the normal tissue regeneration after applying PDT [6]. Yet PDT appeared to have its own limitations that vary from the penetration limit of the light itself, skin sensitivity and the sub therapeutic dose accumulated in the cancerous tissue. These drawbacks hindered the development of this kind of treatment in front of other conventional approaches. Recently, the development of new PS, light sources as well as drug delivery machinery and the activation of the host immune response are all factors that can play an enormous role to increasing the chances of PDT to be applied either alone or combined in the effective treatment of cancer disease. Recently, silver nanoparticles (Ag NPs) have got an increased interest especially after showing in a previously published work the possibility of using them as PS. Generally, Ag NPs have distinctive characteristics and applications from ink-jet printing to antimicrobial usage_and was found to affect neurons and undifferentiated cells [7-9]. In our previous study Ag NPs have been shown to have cytotoxic effect after irradiation with laser light on two cancer lines namely MCF-7 and A549 cells [10]. The mechanism is believed to be photochemical rather than photo-thermal according to the chosen laser parameters. That means that reactive oxygen species have been produced in the vicinity of those cell lines that were responsible for their death.

The aim of the present work was to study closely how Ag NPs mediate their cytotoxicity and if they affect the cells' DNA or not. Hence a better understanding of their way of action can help in future development in such nanostructures and their applications in PDT alone and/or with combined chemotherapy.

\section{Materials and Methods}

\section{Cell culture}

Lung cancer (A549) cell lines were originated from the American type Cell Culture (ATCC; Manassas, VA, USA) (ATCC HTB-53). Cell

*Corresponding author: Ahmed El-Hussein, The National Institute of Laser Enhanced Science, Cairo University, Egypt, Tel: 202 35715882; E-mail: a.el-husseiny@niles.edu.eg

Received December 23, 2015; Accepted Janaury 11, 2016; Published January 20, 2016

Citation: El-Hussein A (2016) Study DNA Damage after Photodynamic Therapy using Silver Nanoparticles with A549 cell line. J Nanomed Nanotechnol 7: 346. doi:10.4172/2157-7439.1000346

Copyright: ( 2016 El-Hussein A. This is an open-access article distributed under the terms of the Creative Commons Attribution License, which permits unrestricted use, distribution, and reproduction in any medium, provided the original author and source are credited. 
Culture growth media were manufactured by Gibco (Thermo Fisher Scientific, Waltham, MA, USA). A549 cancerous cells were cultured in Roswell Park Memorial Institute media that was supplemented with $10 \%$ Fetal Bovine Serum and $1 \%$ penicillin/streptomycin/fungizone (GE Healthcare Bio-Sciences Corp., Piscataway, NJ, USA) with $5 \% \mathrm{CO}_{2}$ and at $37^{\circ} \mathrm{C}$. Cells were washed by Hank's balanced salt solution (HBSS; Thermo Fisher Scientific) upon reaching confluency and trypsinized by TrypLE ${ }^{\mathrm{TM}}$ Express (Gibco ${ }^{\oplus}$; Thermo Fisher Scientific). Cells were seeded with a concentration of $3 \times 10^{5}$ in culture dishes of $3.3 \mathrm{~cm}$ diameter where they were left 4 hours to attach. Then $3.23 \mathrm{mg} / \mathrm{ml}$ of Ag NPs were introduced to those attached cells and left overnight. Laser irradiations with $20 \mathrm{~J} / \mathrm{cm}^{2}$ were performed at dark through an optical fiber at room temperature where the laser spot size covered exactly the cells monolayer in the culture plates. The parameters of the used laser were summarized in our previous work [10] and summarized in Table 1. The Ag NPs were synthesized at the Institute of Photonic Technology in Jena, Germany. The used Ag NPs have been described in an earlier work [10] where the stock solution was of a concentration $80.884 \mathrm{mg} / \mathrm{ml}$.

\section{Laser induced breakdown spectroscopy}

The Ag NPs that were used in the current study have an absorption peak at $630 \mathrm{~nm}$ which is the not in the usual absorption spectrum of silver. Laser induced breakdown spectroscopic (LIBS) technique was used to confer more validation about the identity of the used nanoparticles. It is an elemental quazi invasive tool with minimal sample preparation [11]. The setup is described before by Ahmed et al. [12].

\section{Experimental setup}

The current experiment had the setup where cell cultures were divided into four study groups: The first one was used as a control where cells were cultured normally in its nourishing media and with its $5 \% \mathrm{CO}_{2}$ supplement; the second group was treated similarly but irradiated with a light dose of $20 \mathrm{~J} / \mathrm{cm}^{2}$; the third group was treated as group one but with the addition of $3.23 \mathrm{mg} / \mathrm{ml} \mathrm{Ag} \mathrm{NPs}$; the last group was the PDT group as the cells of this group had both $3.23 \mathrm{mg} / \mathrm{ml} \mathrm{Ag}$ NPs and laser irradiation at $20 \mathrm{~J} / \mathrm{cm}^{2}$.

\section{ROS evaluation}

Intracellular reactive oxygen species (ROS) was estimated in all the four experimental groups where $1 \times 10^{5}$ of A549 cells were plated in 96 well plates accordingly and washed with PBS twice. The cells were then incubated with $10 \mathrm{uM}$ 5-(and-6)-chloromethyl-2, 7-dichlorodihydrofluorescein diacetate acetyl ester (CM-H2DCFDA)

\begin{tabular}{|c|c|}
\hline Parameters & Diode laser \\
\hline Manufacturer & Oriel Corporation \\
\hline Wavelength & $636 \mathrm{~nm}$ \\
\hline Wave emission & Continuous \\
\hline Spot size & $9.1 \mathrm{~cm}$ \\
\hline Output power & $88 \mathrm{~mW}$ \\
\hline Power density & $10.288 \mathrm{~mW} / \mathrm{cm}^{2}$ \\
\hline \multirow{2}{*}{ Fluences } & $10 \mathrm{pun}$ \\
\hline \multirow{2}{*}{ Irradiation times } & $15 \mathrm{Jim}$ \\
\cline { 2 - 2 } & $20 \mathrm{~J} / \mathrm{cm}$ \\
\hline & 16 minutes, $11 \mathrm{~seconds}$ \\
\hline
\end{tabular}

Table 1: Parameters of the lasers used in this study. dye at $37^{\circ} \mathrm{C}$ for 1 hour. Where after, the Fluorescence intensity was measured at $530 \mathrm{~nm}$ via the plate reader.

\section{Mitochondrial membrane potential measurements}

Moreover, the mitochondrial membrane potential, $\Delta \Psi \mathrm{m}$ was needed to be investigated. This parameter gets its importance since it can be used as cell health indicator. JC-1 mitochondrial membrane potential assay kit (Cayman chemical, Michigan, USA), Zeiss confocal microscope and the fluorescence plate reader were used in the current research for such purpose. The physics of that kit relies on thelipophilicity of JC-1 and its cationic nature that can enter the mitochondria in a selective manner where it forms complex called J-aggregates whose color is green upon healthy conditions and otherwise reversibly change its color into red. The assay can be described briefly as follow; The cells from the four experimental groups were plated in 96 well plates at $1 \times$ $10^{5} \mathrm{cells} / \mathrm{ml} .100 \mu \mathrm{l} / \mathrm{ml}$ of JC- 1 staining solution is added to each well and mixed gently. The pates were then incubated for 15-30 minutes at $37^{\circ} \mathrm{C}$ and $5 \% \mathrm{CO}_{2}$. The plates were then read using excitation/emission $540 / 570 \mathrm{~nm}$ for healthy cells or 485/535 nm for apoptotic conditions and imaged using Zeiss multi lasers confocal microscope.

\section{Comet assay}

Single cell gel electrophoresis was used for the estimation of DNA damages that may be caused through the usage of Ag NPs as a PS in PDT treatment of A549 cell line. The assay was explained in details in an earlier work [13] where a visual assessment to the relative tail intensity using Zeiss upright microscope associated with a CCD camera was done, by sorting 100 comets into classes from 0 (no detectable tail) to 4 (large tail, minimal head), giving an overall damage score of $0-400$.

All sets of experiments were repeated four times with duplicate replications of each assay. Origin pro 8 was used as software for performing the one way statistical analysis to get all the mean, standard deviation, and standard error values. P-values were designated for the statistical significance between the control groups and the PDT treated groups with Ag NPs.

\section{Results and Discussion}

LIBS technique confirmed the elemental constituent of the synthesized nanoparticles to be silver. According to the national institute of standards and technology (NISTA), the spectral line which is at $328.2 \mathrm{~nm}$ should be to silver element as shown in Figure 1.

The current study proves that PDT mediated cytotoxicity using Ag NPs was found to be via the generation of intracellular ROS as shown in Figure 2. Yet the dosage and exposure time are needed to be further studied for their importance and complexity. It is apparent from the
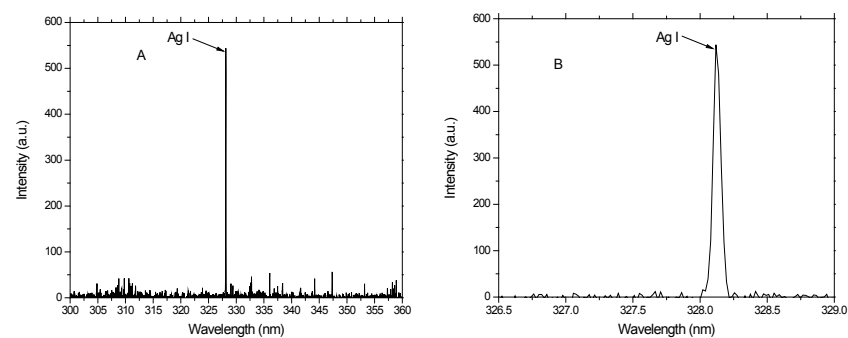

Figure 1: LIBS Spectra showing the silver spectral line with high intensity for the synthesized nanoparticles confirming the existence of silver. 


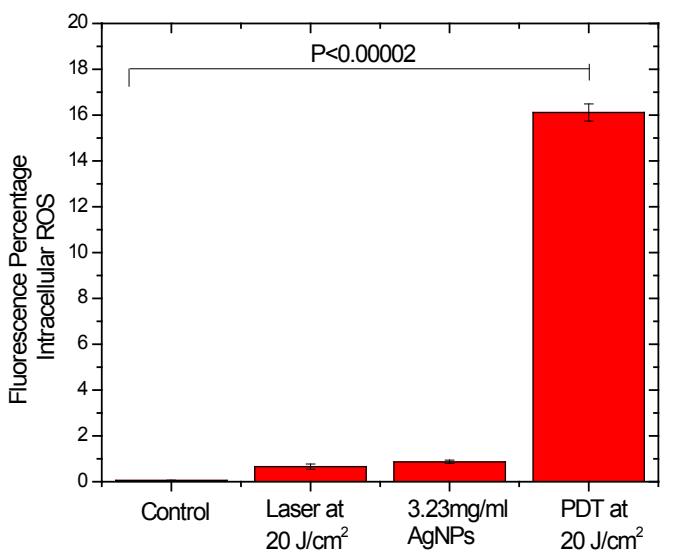

Figure 2: Different experimental groups stained with Fluorescent 5-(and-6)chloromethyl-2,7 dichlorodihydrofluoresce in diacetate acetyl ester showing the generation of intracellular ROS in the PDT group with significance $p<0.00002$ compared to the control group.

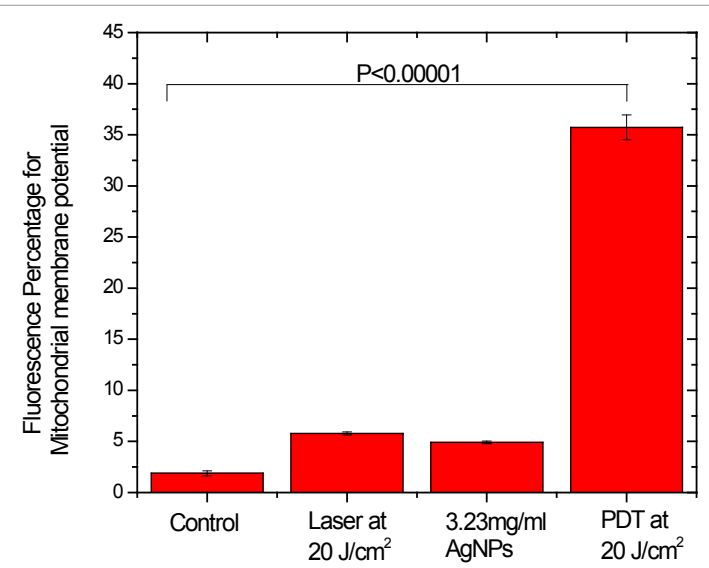

Figure 3: Different experimental groups stained with JC-1 fluorescent dye showing the significant change and hence the mitochondrial membrane potential in the PDT group compared to the control one where $p<0.0001$.

figure that the Ag NPs and the laser irradiation alone resulted in nonsignificant amount of ROS compared to the

control group. On the other hand the PDT group generated intracellular biphasic ROS in a significant yield compared to the control group where $\mathrm{p}<0.00002$.

Many studies have reported the generation of such ROS in many cell lines due to the introduction of nanoparticles like Mukherjee et al. [14] and Bhattacharya et al. [15] Ag NPs can be one of the most prominent candidates in nanomedicine since they are believed to have a big role in the cellular oxidative stress which consequently results in ROS production. The cytotoxicity of Ag NPs have shown by many research centers to be size and shape dependent [16-18]. As long as the size of the Ag NPs decreases, their exposed surface area that can be involved in the biochemical interactions with the cells is increased and hence their cellular effects are elevated by several folds post laser irradiation. The size of the used Ag NPs in the current study was to be $27 \mathrm{~nm}$ and of spherical shape that rendered them to be very relevant and preferable in such kind of applications.

A significant increase in the mitochondrial membrane potential $(\triangle \Psi \mathrm{m})$ in the PDT group was observed when compared to the control group $(\mathrm{p}<0.00001)$ as shown in Figure 3 during the tracing of $(\Delta \Psi \mathrm{m})$ in the different experimental groups. Noting that there was no such drastic increase in the mitochondrial membrane potential for the Ag NPs group only nor the laser light only group confirming the viability study that has been shown in our previous study [10] where either laser light alone or Ag NPs alone have any toxic effects on A549 cells with the used light intensity and concentration respectively.

Confocal microscopic imaging added strength to the above results by showing the green monomer form of the JC-1 fluorescent dye in the case of the PDT experimental group where the other experimental groups have shown the healthy red J aggregates as a result of the complex formation in the mitochondria as shown in Figure 4.

Despite the existence of many pathways for the generation of ROS, one can adopt the mitochondrial generation of ROS pathway via the electron transport chain mechanism [19]. This can be attributed to the correlation between the significance increase in the intracellular ROS and the mitochondrial membrane potential of the PDT group compared to the controls.

Studying the genotoxicity that may be caused by Ag NPs in the PDT treatment of A549 cells using the comet assay showed a significant DNA fragmentation in the PDT group compared to the control group where $p<0.0001$ as shown in Figure 5. The increased DNA damage in the PDT group matches perfectly with the previously published results [10] where the viability percentage and cellular proliferation significantly dropped in the PDT treated cells as well as increased levels of Lactate dehydrogenase (LDH) which is an indication of increased cytotoxicity.

There is not yet a confirmation about the sole pathway of the mitochondrial ROS generation in the Ag NPs mediating cytotoxicity. As there might be other cell organelles and pathways that could be

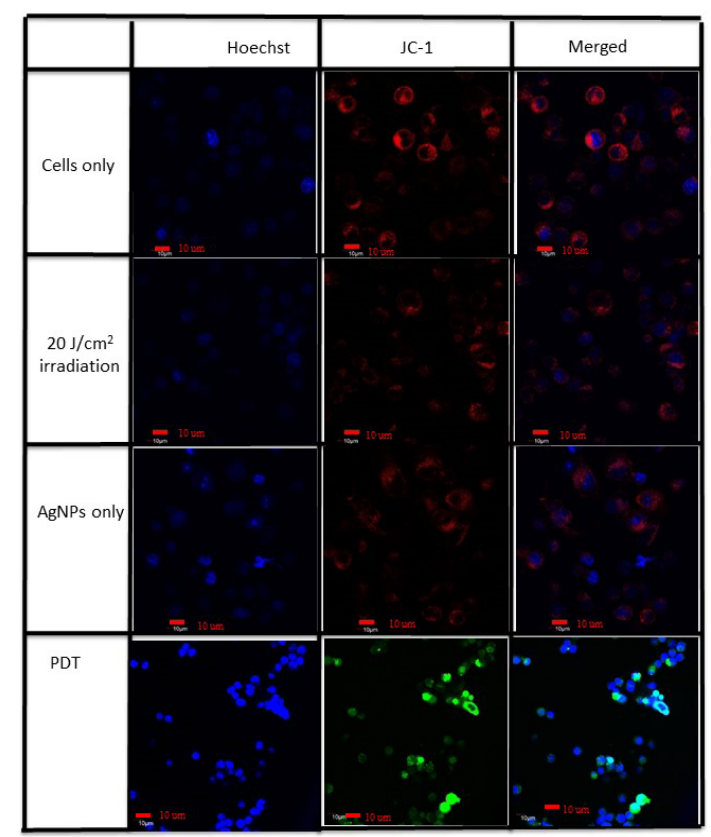

Figure 4: Confocal imaging to control, Ag NPs only and laser of $20 \mathrm{~J} / \mathrm{cm}^{2}$ only show the healthy red J-aggregates in the mitochondria while the PDT group shows the green $\mathrm{J}$-monomer due to the increased mitochondrial membrane potential. 


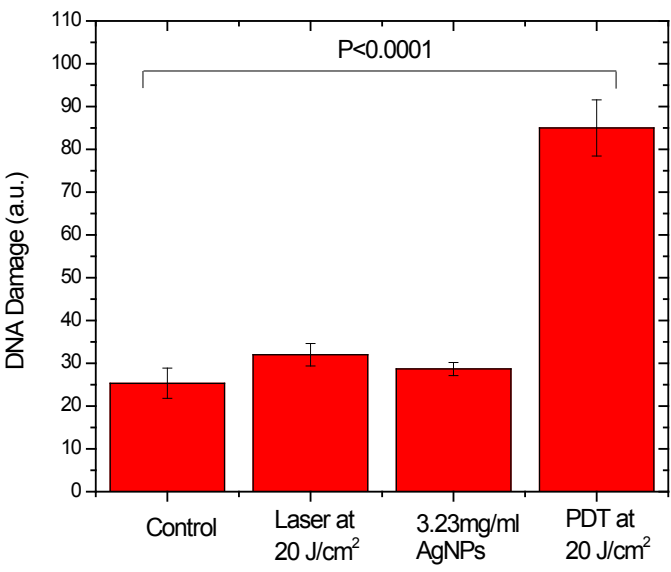

Figure 5: Comet assay measuring the DNA for the four experimental groups of A549 cancer cells, showing significant DNA damage after PDT treatment compared to the control group $(p<0.0001)$

involved in these reactions with other probabilities of the presence of increased post oxidative stress after intracellular ROS generation and DNA fragmentation. O'Rourke has investigated the relationship between the change of the mitochondrial membrane potential and ROS generation, where he concluded that the rate of activation of the $\mathrm{K}+$ ion for the oxidative phosphorylation is elevated by increasing the mitochondrial membrane potential and hence increased level of ROS [20]. One proposed mechanism could be estimated from the overall data and from other recent researches [21] that the silver ions which were generated after laser irradiation can intervene with the DNA replication after the induction of ROS and eventually cell death. The produced silver cations may act as oxidative entities by capturing electrons and hence can reduce cellular ATP contents and consequently can be described as cell death effectors through enhancing of ROS production [22]. There are two main pathways of apoptosis by PDT, which are extrinsic (cytoplasmic) or intrinsic (mitochondrial pathway). The latter pathway is initiated when the mitochondrial membrane becomes more permeable and release of Cytochrome $\mathrm{C}$ into the cytosol. Where it interacts with apoptotic protease activity factor and dATP to form apoptosome. The latter leads to the activation of Caspase- 9 that in turn activates Caspase- 3 where it activates the rest of caspase cascade. Eventually the process ends by the cleavage of PARP (poly ADP-ribose polymerase) and DNA fragmentation. In our previous work, during the characterizing the Zeta potential of the used Ag NPs [10], it was found to have very small value that may results in their aggregation upon protein adsorption on their surface $[23,24]$. Cho et al. have discussed the effect of Zeta potential and the nanoparticles' size as a crucial factor on their agglomeration and hence in inducing cellular response and specified a threshold size limit of $50 \mathrm{~nm}$ [25]. The current work showed no genotoxicity of the Ag NPs by itself, but after their light absorption they generate ROS with the change of mitochondrial membrane potential that can leads to a serious of reactions that eventually leads in the absence of antioxidant protection to DNA damage and apoptosis [26]. Nevertheless, ROS can be induced via other organelles and pathways, as Golgi, endosomes and lysosomes after cellular exposure to other NPs [26].

\section{Acknowledgement}

Acknowledgements are extended to Professor Wolfgang Fritzsche and his research team, Nano Biophotonics Department, Institute of Photonic Technology (IPHT), Jena, Germany for the synthesis and supply of Ag NPs.

\section{Conflict of Interests}

The author confirms the absence of any conflict of interests regarding the current work.

\section{References}

1. El Hussein A, Mfouo-Tynga I, Abdel-Harith M, Abrahamse H (2015) Comparative study between the photodynamic ability of gold and silver nanoparticles in mediating cell death in breast and lung cancer cell lines. J Photochem and Photobiol B 153: 67-75.

2. Jemal A, Bray F, Center MM, Ferlay J, Ward E, et al. (2011) Global cancer statistics. CA Cancer J Clin 61: 69-90.

3. Kato H (1998) Photodynamic therapy for lung cancer - A review of 19 years experience. J Photochem and Photobiol B 42: 96-99.

4. Simone CB, Friedberg JS, Glatstein E, Stevenson JP, Sterman DH, et al. (2012) Photodynamic therapy for the treatment of non-small cell lung cancer. $\mathrm{J}$ Thorac Dis 4: 63-75.

5. Agostinis P, Berg K, Cengel KA, Foster TH, Girotti AW, et al. (2011) Photodynamic therapy of cancer: an update. CA Cancer J Clin 61: 250-281.

6. Su J, Zhang J, Liu L, Huang Y, Mason RP (2008) Exploring feasibility of multicolored $\mathrm{CdTe}$ quantum dots for in vitro and in vivo fluorescent imaging. $\mathrm{J}$ Nanoscie and Nanotechnol 8: 1174-1177.

7. Marambio-Jones C, Hoek EMV (2010) A review of the antibacterial effects of silver nanomaterials and potential implications for human health and the environment. J of Nanoparticle Reser 12: 1531-1551.

8. Barbasz A, Oćwieja M, Barbasz J (2015) Cytotoxic activity of highly purified silver nanoparticles sol against cells of human immune system. Appl. Biochem. Biotechnol 176: 817-834.

9. Mfouo-Tynga, I, Hussein A, Harith M, Abrahamse H (2014) Photodynamic Ability of Silver Nanoparticles in Inducing Cytotoxic Effects in Breast and Lung Cancer Cell Lines. Int J of Nanomed 9: 3771-3780.

10. El-Hussein A, kassem AK, Ismail H, Harith MA (2010) Exploiting LIBS as spectrochemical analytical technique in diagnosis of some types of human malignancy. Talanta 82: 495-501.

11. El Hussein A, Marzouk A, Harith MA (2015) Discriminating crude oil grades using laser-induced breakdown spectroscopy. Spectrochim Acta B 113: 93-99.

12. El-Hussein A, Harith MA, Abrahamse H (2012) Assessment of DNA damage after photodynamic therapy using a metalphthalocyanine photosensitizer.

13. Mukherjee SP, Lyng FM, Garcia A, Davoren M, Byrne HJ, et al. (2010) Mechanistic studies of in vitro cytotoxicity of poly (amidoamine) dendrimers in mammalian cells. Toxicol and App Pharma 248: 259-268.

14. Bhattacharya K, Pratap C, Nahaa C, Naydenovad I, Mintovae S, et al. (2012) Reactive oxygen species mediated DNA damage in human lung alveolar epithelial (A549) cells from exposure to non-cytotoxic MFI-type zeolite nanoparticles. Toxicol Lett 215: 151-160.

15. Carlson C, Hussain SM, Schrand AM, Braydich-Stolle LK, Hess KL, et al. (2008) Unique cellular interaction of silver nanoparticles: size-dependent generation of reactive oxygen species. The Journal of Physical Chemistry B 112: 1360813619.

16. Kim TH, Kim M, Park HS, Shin US, Gong MS, et al. (2012) Size-dependent cellular toxicity of silver nanoparticles. J of Biomed Mat Res Part A 100: 10331043.

17. Morones JR, Elechiguerra JL, Camacho A, Holt K, Kouri JB, et al. (2005) The bactericidal effect of silver nanoparticles. J Nanotechnology 16: 2346-2353.

18. McLennan HR, Esposti MD (2000) The contribution of mitochondrial respiratory complexes to the production of reactive oxygen species. J Bioenergetics and Biomembranes 32: 153-162.

19. O’Rourke B (2007) Mitochondrial ion channels. Annual Review of Physiology 69: $19-49$.

20. Kreuter J, Gelperina S (2008) Use of nanoparticles for cerebral cancer. Tumori 94: 271-277.

21. AshaRani PV, Low KahMun G, Hande MP, Valiyaveettil S (2009) Cytotoxicity and genotoxicity of silver nanoparticles in human cells. ACS Nano 23: 279-290. 
Citation: El-Hussein A (2016) Study DNA Damage after Photodynamic Therapy using Silver Nanoparticles with A549 cell line. J Nanomed Nanotechnol 7: 346. doi:10.4172/2157-7439.1000346

Page 5 of 5

22. Gray JJ (2004) The interaction of proteins with solid surfaces. Current Opinion in Structural Biology 14: 110-115.

23. Lynch I, Cedervall T, Lundqvist M, Cabaleiro-Lago C, Linse S (2007) The nanoparticle-protein complex as a biological entity: a complex fluids and surface science challenge for the $21^{\text {st }}$ century. Advances in Colloid and Interface Science 134: 167-174

24. Cho EC, Zhang Q, Xia Y (2011) The effect of sedimentation and diffusion on cellular uptake of gold nanoparticles. Nature Nanotechnology 6: 385-391.
25. Halliwell B, Whiteman M (2004) Measuring reactive species and oxidative damage in vivo and in cell culture: how should you do it and what do the results mean? British Journal of Pharmacology 142: 231-255.

26. Jiang Z, Hu Z, Zeng L, Lu W, Zhang H, et al. (2011) The role of the Golg apparatus in oxidative stress: is this organelle less significant than mitochondria? Free Radical Biology and Medicine 50: 907-917. 